\title{
Acolhimento aos familiares de vítimas de óbitos por
}

\section{causas externas}

\section{Family hosting of victims' death external causes}

\author{
Wbiratan de Lima Souza ${ }^{1} \bullet$ Cristina Lavoyer Escudeiro² $\bullet$ Geisiane de Souza Santos ${ }^{3} \bullet$ Lucimere Maria dos Santos $^{4} \bullet$ \\ Eliane Ramos Pereira ${ }^{5}$ Rose Mary Costa Rosa Andrade Silva ${ }^{6}$
}

\begin{abstract}
RESUMO
Os agravos que contemplam as causas externas, anualmente, matam mais de 5 milhões de pessoas em todo o mundo, o que representa cerca de $9 \%$ da mortalidade mundial. Este estudo objetivou descrever a experiência vivenciada pela equipe no acolhimento aos familiares de vítimas de óbitos por causas externas em um hospital de referência em Alagoas. Trata-se de um relato de experiência vivenciado no decorrer das práticas profissionais em um hospital de referência em trauma em Alagoas. Desde da implantação do Hospital em 2003 aos dias atuais, várias transformações foram acontecendo na prática hospitalar, como demanda de vítimas de acidentes ou violências, bem como ocorrências de óbitos foram aumentando. Simultaneamente a todas essas mudanças, vários questionamentos iam sendo feitos pelos próprios profissionais e familiares referentes à condução do corpo após o óbito. A partir da análise desta realidade, evidenciou-se a necessidade de definição de um fluxograma, com participação efetiva da equipe interdisciplinar, para facilitar a condução do corpo após o óbito. Portanto, o trabalho da equipe interdisciplinar no acolhimento aos familiares de vítimas de óbitos por causas externas caracterizou-se como uma estratégia de aperfeiçoamento pessoal, profissional e teórico/prático-científico, possibilitando redução do tempo para liberação do corpo da Unidade Hospitalar para o Instituto Médico Legal e, posteriormente, velório e sepultamento.
\end{abstract}

Palavras-chave: Comunicação Interdisciplinar; Acolhimento; Morte; Relações Profissional-Família; Causas Externas.

\begin{abstract}
The external causes' aggravation annually kill more than 5 million people worldwide, representing about $9 \%$ of global mortality. This study aimed to describe the experience lived by the reception staff to family victims' external causes in a referral trauma specialized hospital in Alagoas. Since the deployment - in 2003 - until today, several changes were happening in hospital practice, as demand for victims of accidents or violence and occurrences' deaths were being increased. Simultaneously, with all these changes, many questions were being made by professionals themselves and their families regarding the conduct of the body after death. From the analysis of this reality, it showed the indispensability on defining a flow chart, with the effective participation of an interdisciplinary team, to facilitate body conduction after death. Therefore, the work of the interdisciplinary team in welcoming these death's parents characterized as personal development strategy, professional and theoretical / practical-scientific, enabling reduced time to release the body from the Hospital Unit to Forensic Institute and then wake and burial.
\end{abstract}

Keywords: Interdisciplinary Communication; User Embracement; Death; Professional-Family relations; External Causes.

Enfermeiro. Mestrando do Programa de Mestrado Profissional em Enfermagem Assistencial (MPEA) da Escola de Enfermagem Aurora de Afonso Costa (EEAAC) da Universidade Federal Fluminense (UFF). Professor do Centro Universitário Tiradentes (UNIT-Maceió). Professor da Universidade Estadual de Ciências da Saúde de Alagoas (UNCISAL). Professor substituto da Universidade Federal de Alagoas (UFAL-Maceió). Enfermeiro Assistencial do Hospital de Emergência 1 Dr. Daniel Houly (HEDH). Membro do Núcleo de Estudo e Pesquisa em Cidadania e Gerência e Enfermagem (NECIGEN). Maceió - AL, Brasil. E-mail: wbiratansouza@yahoo.com.br.

${ }^{2}$ Enfermeira. Doutora em Enfermagem. Coordenadora do curso de Graduação em Enfermagem da Escola de Enfermagem Aurora de Afonso Costa (EEAAC) da Universidade Federal Fluminense (UFF). Docente Permanente do MPEA (EEAAC/UFF). Membro do NECIGEN. Niterói - RJ. Brasil. E-mail: cristinalescudeiro@gmail.com.

3 Enfermeira. Mestranda do Programa de Mestrado Profissional em Enfermagem Assistencial (MPEA) da Escola de Enfermagem Aurora de Afonso Costa (EEAAC) da Universidade Federal Fluminense (UFF). Enfermeira do Hospital Estadual Roberto Chabo. Membro do Núcleo de Estudo e Pesquisa em Cidadania e Gerência e Enfermagem (NECIGEN). Saquarema - RJ, Brasil. E-mail: grisianesantos10@hotmail.com.

${ }^{4}$ Enfermeira. Mestranda do Programa de Mestrado Profissional em Enfermagem Assistencial (MPEA) da Escola de Enfermagem Aurora de Afonso Costa (EEAAC) da Universidade Federal Fluminense (UFF). Enfermeira do Instituto Nacional do Câncer (INCA - Ambulatório de Oncologia Clínica e Hematologia). Duque de Caxias - RJ, Brasil. E-mail: lucimere_santos@hotmail.com.

${ }^{5}$ Enfermeira. Pós-Doutorado em Enfermagem. Coordenadora e Docente do Programa de Mestrado Profissional em Enfermagem Assistencial (MPEA), Professora do Curso de Graduação (EEAAC/UFF). São Gonçalo - RJ. Brasil. E-mail: elianeramos.uff@gmail.com.

${ }^{6}$ Enfermeira, Psicóloga e Filósofa. Doutora em Enfermagem. Vice - Coordenadora do Programa de Mestrado Ensino na Saúde (MPES) da Escola de Enfermagem Aurora de Afonso Costa (EEAAC) da Universidade Federal Fluminense (UFF). Docente Permanente do MPEA (EEAAC/UFF). Barra da Tijuca - RJ. Brasil. E-mail: roserosauff@gmail.com. 


\section{INTRODUÇÃO}

Os agravos que contemplam as causas externas, anualmente, matam mais de 5 milhões de pessoas em todo o mundo, o que representa cerca de $9 \%$ da mortalidade mundial. Causam, também, danos a outros milhões de sobreviventes, gerando repercussões de hospitalizações, bem como atendimentos ambulatoriais e de emergência'-2.

Em 2011, no Brasil, dados do Ministério da Saúde (MS) mostraram que as causas externas vitimaram cerca de 145 mil pessoas, correspondendo à terceira maior causa de morte no país ( $12 \%$ do total), e foram responsáveis por cerca de 1 milhão de internações (aproximadamente 9\% do total), sendo a quinta causa de internações no Sistema Único de Saúde (SUS), e, vale ressaltar, que as vítimas que não necessitaram de internação não fazem parte destes dados estatísticos ${ }^{3}$.

No Brasil, o coeficiente padronizado de mortalidade por causas externas é muito maior entre homens (178 por 100 mil habitantes) do que entre mulheres (24 por 100 mil habitantes), sendo maiores entre homens mais jovens dentre 20 a 29 anos, em todas as regiões, e diminuindo com a idade, dando ênfase aos valores maiores nas regiões Norte e Nordeste ${ }^{4}$.

Os acidentes e violências são considerados como um conjunto de agravos que podem, de acordo com a vulnerabilidade e fatores de riscos comportamentais, configurar-se ou não no óbito, isto é, passível e previsível de prevenção, e configuram-se como um problema de saúde pública ${ }^{5-6}$.

Neste grupo de causas externas, incluem-se as lesões provocadas por eventos de transporte, suicídios, homicídios, agressões, envenenamentos, quedas, afogamentos, queimaduras, lesões por deslizamento ou enchente, e outras ocorrências provocadas por circunstanciais ambientais. Este conjunto de agravos está inserido na Classificação Internacional de Doenças - CID 10, catalogado no eixo das causas externas (capítulo XX da CID 10), entre outros (capítulo XIX da CID 10) ${ }^{3-7}$.

Frente ao grau de relevância no contexto social, no que se remete às repercussões da mortalidade decorrente desses agravos, para emissão de dados confiáveis referentes à mortalidade, foi implantado no Brasil, na década de 1970 o Sistema de Informações de Mortalidade (SIM), no intuito de apresentar de forma gradativa e aprimorada dados mais precisos, gerando, assim, uma qualidade na informação ${ }^{8}$.

Quanto à causa da morte, ainda hoje, as informações do SIM são afetadas pela presença de causas mal definidas (Capítulo XVII da Classificação Estatística Internacional de Doenças e Problemas Relacionados à Saúde - Décima Revisão (ID 10), assim como dos diagnósticos incompletos, que se constituem em causas residuais de Capítulos de causas bem definidas ${ }^{8,9}$.

Atualmente, no Brasil, as investigações de óbitos por causas mal definidas que têm como agravos acidentes e violências, são de responsabilidade do Instituto Médico Legal (IML), que tem como finalidade tentar minimizar tais situações, emitindo laudos mais concretos e exatos sobre a real causa do óbito, corroborando uma informação mais confiável e precisa ${ }^{8,9}$.

Os Hospitais de referência em Trauma em Alagoas seguem o mesmo fluxo do país, que, em casos de óbitos por causas de acidentes e violência, emite-se uma guia de transferência, na qual o corpo deve ser encaminhado para o IML para emissão de um laudo e, consequentemente, da Declaração de Óbito (DO), com a definição da causa ${ }^{8,9}$.

Entretanto, sabe-se que, nos serviços de saúde, muitas vezes não existe um acolhimento humanizado aos familiares que passam por um momento crítico e de desestabilidade emocional devido à perda de um ente querido, no que se refere à concessão de informações claras e concisas sobre as etapas a serem percorridas, desde a liberação do corpo para o velório até o sepultamento, facilitando, assim, o caminho a ser percorrido pelos familiares ${ }^{10}$.

Diante do exposto, o objetivo deste relato é descrever a experiência vivenciada pela equipe interdisciplinar no acolhimento aos familiares de vítimas de óbitos por causas externas em um hospital de referência em Alagoas.

\section{MÉTODO}

Trata-se de um relato de experiência vivenciado no decorrer das práticas profissionais em um hospital de referência em trauma em Alagoas, cuja trajetória histórica se deu no período de 2009 a 2015, no entanto, este foi descrito em 2015.

Alagoas, um estado brasileiro, composto por 102 municípios, tendo como principais referências Maceió (Capital) e a segunda maior cidade do estado, Arapiraca; cada cidade foi intitulada como polo afim de descentralizar as ações e os serviços para os outros municípios. Da mesma forma, foram criados dois Institutos Médico Legal - IMLs - um em cada cidade, com intuito de favorecer laudos mais rápidos e precisos sobre os óbitos por acidentes e violências relacionadas, em geral, por causas externas.

De acordo com dados do Instituto Brasileiro de Geografia e Estatística (2015), em 2015,Alagoas possui uma população estimada de 3.300 .938 habitantes, sendo destes 1.013.773 habitantes em Maceió, e 231.053 habitantes em Arapiraca, isto é, praticamente $50 \%$ da população do estado habitam nessas duas cidades ${ }^{11}$.

Diante disso, o estado implantou dois grandes serviços públicos de saúde de referência, o Hospital Geral do Estado (HGE), localizado na capital alagoana, tendo como referência situações de urgências e emergências clínicas e/ou traumáticas; e, na cidade de Arapiraca, foi implantado o Hospital de Emergência Dr. Daniel Houly (HEDH), referência para a segunda macrorregional em urgências e emergências apenas traumáticas. 


\section{DESCREVENDO A EXPERIÊNCIA}

\section{Breve contexto social, histórico e cultual da instituição}

O Hospital de Emergência Dr. Daniel Houly, situado na cidade de Arapiraca, município de Alagoas que está localizado a 101,87 Km da capital Maceió, na região agreste, e possui uma extensão territorial de $351,48 \mathrm{Km}^{2}$ de acordo com a Associação dos Municípios Alagoanos (AMA). Arapiraca ficou conhecida, nos anos 70, como a "Capital do Fumo" por ser um dos maiores produtores de tabaco do país ${ }^{11}$. Desde sua implantação em 18 de julho de 2003, o HEDH é uma instituição pública especializada em atendimentos de urgências e emergências traumáticas. Atende a 56 dos 102 municípios de Alagoas, em um universo de aproximadamente 1.100 .000 habitantes das regiões do Sertão, Agreste e Baixo São Francisco, buscando atender à grande demanda de usuários, tendo em vista a grande proliferação de acidentes de trânsito e a boa localização do município.

Diante disso, vale considerar que entender o desenvolvimento da complexa organização denominada hospital e, ainda, como se dá o processo de configuração de um modelo de gestão, além de considerar o enfoque centrado no cliente pode proporcionar aos administradores brasileiros não um modelo a ser meramente copiado sem uma reflexão de sua aplicabilidade, em cada realidade, mas, sim, um norte de como os hospitais podem chegar a ser empresas proativas e com um modelo de gestão implantado de maneira consciente pelo grupo de poder ${ }^{12}$.

\section{Refletir, conhecer e viver sobre o processo de morte e morrer: transcendendo a forma tradicional do manejo do corpo em óbito}

A equipe interdisciplinar em saúde, de forma direta e/ou indireta, participa do processo de morte dos pacientes graves, ou mesmo do óbito súbito, principalmente com o sofrimento dos familiares durante as visitas diárias ao paciente grave, em fase terminal, ou no momento da notícia do óbito. 0 enfermeiro e o médico são os primeiros profissionais a identificarem o óbito, por serem os responsáveis pela assistência direta ao paciente crítico. Ao médico lhe é dado a incumbência de notificar o óbito ${ }^{13}$; ao enfermeiro e equipe de enfermagem, identificar sinais e sintomas de instabilidade hemodinâmica que possam levar ao óbito, bem como preparar o corpo após o óbito. Diante do óbito estabelecido, vale ressaltar a importância do psicólogo e do assistente social. O primeiro é acionado para apoiar emocionalmente os familiares, e ao segundo cabe a responsabilidade de acompanhar todas as etapas necessárias com o encaminhamento do corpo ao IML, bem como orientações sobre as etapas da liberação do corpo. Assim, todos os profissionais, contribuem de forma direta ou indireta para o acolhimento aos familiares de vítimas de óbitos por causas externas nesta instituição.
Percebe-se que o impacto das causas externas de morbidades no Brasil é bastante relevante por todas as repercussões ocasionadas, seja no âmbito familiar ou socia ${ }^{14}$. Nesta instituição, após o médico identificar o óbito, é realizado o preenchimento de três vias de um formulário denominado de guia de transferência do IML; o enfermeiro aciona a equipe do serviço social, que se responsabiliza em solicitar a presença da família no hospital, caso esta ainda não esteja no local, para dar seguimento às etapas.

\section{Estratégias implantadas para favorecer o cuidar e o acolher aos familiares de vítimas de óbitos por causas externas}

Desde sua implantação até os dias atuais, várias transformações foram acontecendo na prática hospitalar, como demanda de vítimas de acidentes ou violências, bem como ocorrências de óbitos foram aumentando. Simultaneamente a todas essas mudanças, vários questionamentos iam sendo feitos pelos próprios profissionais e familiares referentes à condução do corpo após o óbito.

Diante disso, ao longo dos anos, a equipe do Serviço Social assumiu a responsabilidade pelo acolhimento e repasse das informações do óbito com orientações aos familiares quanto aos procedimentos de encaminhamento ao IML e liberação do corpo.

A partir da análise desta realidade, evidenciouse a necessidade de definição de um fluxograma, com participação efetiva da equipe interdisciplinar, para facilitar a condução do corpo após o óbito, favorecendo a humanização em todas as etapas da vida, incluindo a morte, principalmente para com os familiares em momento de grande emoção, descrito em ANEXO 1 (FLUXOGRAMA DE CONDUÇÃO DO CORPO APÓS O ÓBITO NO HEDH).

\section{CONCLUSÃO}

0 trabalho da equipe interdisciplinar no acolhimento aos familiares de vítimas de óbitos por causas externas em um hospital de referência em Alagoas caracterizou-se como uma estratégia de aperfeiçoamento pessoal, profissional e teórico/prático-científico, possibilitando redução do tempo para liberação do corpo da Unidade Hospitalar para o Instituto Médico Legal e, posteriormente, velório e sepultamento. Além de acréscimos de conhecimento na vida profissional enquanto prática, tornou-se um momento de construção de aquisição de valores sociais, culturais, humanísticos, éticos e logísticos, favorecendo o respeito ao corpo humano, mesmo em óbito, garantindo a aplicabilidade das diretrizes e princípios da humanização no Sistema Único de Saúde - SUS.

Possibilitou,também, lançar luz sobre a vivência de novas estratégias de informação, novas teorias e novas formas de se fazer enfermeiro e integrar uma equipe interdisciplinar na busca de um cuidar e acolher com excelência. Assim, 
a utilização do fluxograma de orientação de atividades, possibilitou à equipe interdisciplinar diminuir a ansiedade dos familiares, bem como facilitou a logística operacional da liberação/transferência do corpo para o IML e posterior sepultamento.

\section{REFERÊNCIAS}

1. Institute for Health Metrics and Evaluation. Global Burden of Diseases, Injuries, and Risk Factors Study. [Internet]. 2013 jun [Acesso em 2015 set 4]. Disponível em: http://www. healthmetricsandevaluation.org/gbd/visualizations/regional.

2. World Health Organization. Violence, injuries and disability. Biennial report 2008-2009. Geneva: World Health Organization Press; 2012.

3. Campos MR, Von Doellinger VDR, Mendes LVP, Costa MDFS, Pimentel TG, de Andrade Schramm JM. Diferenciais de morbimortalidade por causas externas: resultados do estudo Carga Global de Doenças no Brasil, 2008. Cad. Saúde Pública [Internet]. 2015 jan [Acesso em 2015 set 10]; 31(1): 121-36. Disponível em: http://www.scielo.br/pdf/csp/v31n1/pt_0102311X-csp-31-01-00121.pdf.

4. Moura EC, Gomes R, Falcão MTC, Schwarz E, Neves ACM, Santos W. Desigualdades de gênero na mortalidade por causas externas no Brasil, 2010. Ciência \& Saúde Coletiva [Internet]. 2015 [Acesso em 2015 dez 5]; 20(3): 779-88. Disponívcel em: http://www.scielo.br/pdf/csc/v20n3/pt_1413-8123csc-20-03-00779.pdf.

5. Moura LA, Cruz NM, Oliveira LCA, Gurgel PKF, Teixeira MC, Soares WLP. Anos Potenciais de Vida Perdidos por Causas Externas no Rio Grande do Norte, 2003 a 2012.UNOPAR Cient. Ciênc, biol. Saúde [Internet]. 2015 mai [Acesso em 2015 dez 5]; 17(2). Disponível em: http://pesquisa.bvsalud.org/ses/ resource/pt/lil-759595.

6. Matos K, Martins CBG. Mortalidade por causas externas em crianças, adolescentes e jovens: uma revisão bibliográfica. Espaço para a Saúde [Internet]. 2013 dez [Acesso em 2015 set 27]; 14(1/2): 82-93.Disponível em: http://www.uel.br/revistas/ uel/index.php/espacoparasaude/article/view/10480/pdf_7.

7. Tristão KM, Leite FMC, Schmildt ER, Leite EC, Castro DSD, Vilela APM. Mortalidade por causas externas na microrregião de São
Mateus, estado do Espírito Santo, Brasil:tendências de 1999 a 2008. Epidemiologia e Serviços de Saúde [Internet]. 2012 jun [Acesso em 2015 set 13]; 21(2): 305-13. Disponível em: http://scielo.iec.pa.gov. br/scielo.php?script=sci_arttext\&pid=S1679-49742012000200013.

8. Jorge MHPDM, Cascão AM, Reis AC, Laurenti R. Em busca de melhores informações sobre a causa básica do óbito por meio de linkage: um recorte sobre as causas externas em idososEstado do Rio de Janeiro, Brasil, 2006. Epidemiologia e Serviços de Saúde [Internet]. 2012 set [Acesso em 2015 set 13]; 21(3): 407-18. Disponível em: http://scielo.iec.pa.gov.br/scielo. php?script=sci_arttext\&pid=S1679-49742012000300006.

9. Gonsaga RAT, Rimoli CF, Pires EA, Zogheib FS, Fujino MVT, Cunha MB. Avaliação da mortalidade por causas externas. Rev. Col. Bras. Cir. [Internet]. 2012 [Acesso em 2015 out 10]; 39(4): 263-7. Disponível em: http://www.scielo.br/pdf/ rcbc/v39n4/04.pdf.

10. Weykamp JM, Pickersgill CS, Cecagno D, Vieira FP, Siqueira HCHD. Acolhimento com classificação de risco nos serviços de urgência e emergência: aplicabilidade na enfermagem. Rev Rene [Internet]. 2015 jun [Acesso em 2015 dez 18]; 16(3): 327-36. Disponível em: http://www.revistarene.ufc.br/revista/ index.php/revista/article/viewFile/1962/pdf.

11. IBGE, 2015. Disponível em: http://www.ibge.gov.br/estadosat/ perfil.php?sigla=al

12. Merhy EE. O ato de cuidar: a alma dos serviços de saúde. In: Brasil. Ministério da saúde. Secretaria de Gestão do Trabalho e da Educação na Saúde. Departamento de gestão da Educação na Saúde. Ver-SUS Brasil: cadernos de textos. Brasília: Ministério da Saúde; 2004. p. 108-37.

13. Drumond Júnior M, Lira MMTA, Nitrini TMV, Shibao K, Taniguchi M, Bourroul MIM. O novo modelo da declaração de óbito e a qualidade das informações sobre causa externa. Programa de Aprimoramento das Informações de Mortalidade no Município de São Paulo (PRO-AIM). Secretaria Municipal da Saúde. Prefeitura do Município de São Paulo. VI Congresso Brasileiro de Saúde Coletiva. Salvador-Bahia; 2000.

14. Gawryszewski VP, Rodrigues EMS. O impacto das causas externas de morbidades no Brasil, 2003. Sao Paulo Med. J. [Internet]. 2006 [Acesso em 2016 jan 5]; 124(4): 208-13. Disponível em: http://www.scielo.br/scielo.php?script=sci_ arttext \&pid=S1516-31802006000400007. 


\section{ANEXO 1 - FLUXOGRAMA DE CONDUÇÃO DO CORPO APÓS O ÓBITO NO HEDH}

- Atesta o óbito;

- Preenche em três vias a guia de transferência do corpo para o IML ( $1^{\text {a }}$ via (Original) - encaminha para o IML; $2^{\text {a }}$ via - anexa ao prontuário, $3^{\text {a }}$ via - família encaminha para a Polícia Civil solicitando uma requisição de exame cadavérico e a pulseira de identificação (Azul - óbito em Hospital);

- Prepara o corpo;

- Comunica a equipe do Serviço Social para que a família seja informada;

- Familiares chegam no Hospital, a equipe do Serviço Social comunica ao médico

que atestou o óbito para que informe a família;

Serviço

Social

Psicólog

o

- Acionado para dar apoio emocional aos familiares;

- Apoio necessário e as orientações acerca das etapas da transferência do corpo ao IML;

Servico

Social

- Orientar os familiares que devem providenciar cópia e original dos seguintes documentos da vítima: carteira de identidade/RG ou certidão de nascimento ou carteira de trabalho, se houver;

- Munidos dos documentos acima, procurar a Central de Polícia Civil de Arapiraca.

- Familiares procuram a Central da Polícia Civil munido dos documentos de identificação da vítima e de uma guia de transferência preenche uma Requisição de Exame Cadavérico e solicita via telefone que servidores da Polícia Civil levem até o HEDH uma Pulseira de identificação Cadavérica;

- No necrotério do HEDH é inserida na vítima a Pulseira de identificação Cadavérica por um servidor da equipe de padioleiros (maqueiros) acompanhados da equipe da Polícia Civil.

- Comunica a família que já foi feita a identificação do cadáver;

- Contato com o IML para informar da identificação do copro;

- Corpo é direcionado para o Instituto Médico Legal - IML de Arapiraca;

- Parente de primeiro grau deve procurar o IML (Av. Governador Lamenha Filho, Bairro: Jardim Tropical) portando documento de identificação original da vítima,

IML para, assim, ser liberado o corpo, vale ressaltar que o corpo só será liberado depois do laudo do legista, isto é, preenchimento da Declaração de Óbito (DO). 